\title{
EL CUARZO COMO MATERIA PRIMA EN EL VALLE DE HUALFÍN (PROVINCIA DE CATAMARCA, ARGENTINA) DURANTE EL PERÍODO DE DESARROLLOS REGIONALES/INKA
}

\author{
Marina Cecilia Flores \\ CONICET-UNLP. Laboratorio de Análisis Cerámico (FCNyM) calle $64 \mathrm{n}^{\circ} 3$. \\ Laboratorio 18. La Plata (CP 1900). E-mail: marin481@hotmail.com
}

Presentado el: 30/11/2011 - Aceptado 28/03/2012

\section{Introducción}

En los sitios arqueológicos del valle de Hualfín (departamento de Belén, Catamarca) correspondientes al Período de Desarrollos Regionales/Inka (ca. 1000-1535 A.D.) se ha registrado el empleo de diferentes materias primas líticas (obsidiana, basalto, arenisca, cuarzo, entre otras) para la fabricación de instrumentos y/o realización de construcciones. En esta oportunidad se presentan los resultados del análisis tecno-tipológico llevado a cabo sobre los artefactos tallados de cuarzo recuperados en los sitios Campo de Carrizal (Azampay) y Cerro Colorado (La Ciénaga de Abajo).

El cuarzo es un mineral en general incoloro, pero que también aparece en la naturaleza bajo una variada gama de colores, presenta dureza relativa 7 y fractura concoidea, astillosa e irregular (Dana y Hurlbut 1956). Además de estar presente en la mayoría de las rocas ácidas, el cuarzo sólo se presenta en forma pura en pegmatitas y venas hidrotermales, bajo la forma de vetas delgadas o filones potentes que cortan los relieves montañosos. Algunos investigadores (Cortegoso 2008; Gaál 2011) hacen referencia al cuarzo como una materia prima difícil de tallar y formatizar, pero que tiene la ventaja de ser un recurso muy resistente y de larga vida útil, dado que sus filos no se desgranan y se mantienen durante el uso.

En el valle de Hualfín el cuarzo constituye una materia prima de carácter local, como se evidencia a partir de la información geológica (González Bonorino 1972; Ruiz Huidobro 1975) y de las prospecciones realizadas por nosotros en el cerro donde se emplaza el sitio Cerro Colorado, durante las cuales hemos detectado un filón (fuente primaria), siendo por otro lado muy escasa la presencia de cantos rodados observados en el área (fuentes secundarias).

\section{Procedencia de los materiales}

Los materiales de estudio proceden de dos sitios del valle de Hualfín: Campo de Carrizal y Cerro Colorado. El sitio Campo de Carrizal ( $27^{\circ} 19^{\prime}$ S $67^{\circ} 02^{\prime}$ O, 2700 m.s.n.m.) se ubica en el sector noroccidental del valle de Hualfín (figura 1). Se trata de un conjunto de 
núcleos habitacionales conformados por uno o más recintos ubicados a distintas cotas sobre tres espolones enumerados arbitrariamente de oeste a este 1, 2 y 3 (Zagorodny et al. 2007; Wynveldt 2009). Tales núcleos habitacionales se encuentran dispersos entre sistemas de andenes de cultivo y obras de irrigación (acequias y estanques) (Sempé 1981). En recintos ubicados en el segundo y tercer espolón se recuperaron, en excavación, artefactos líticos de diferentes materias primas, locales y no locales (tabla 1 ).

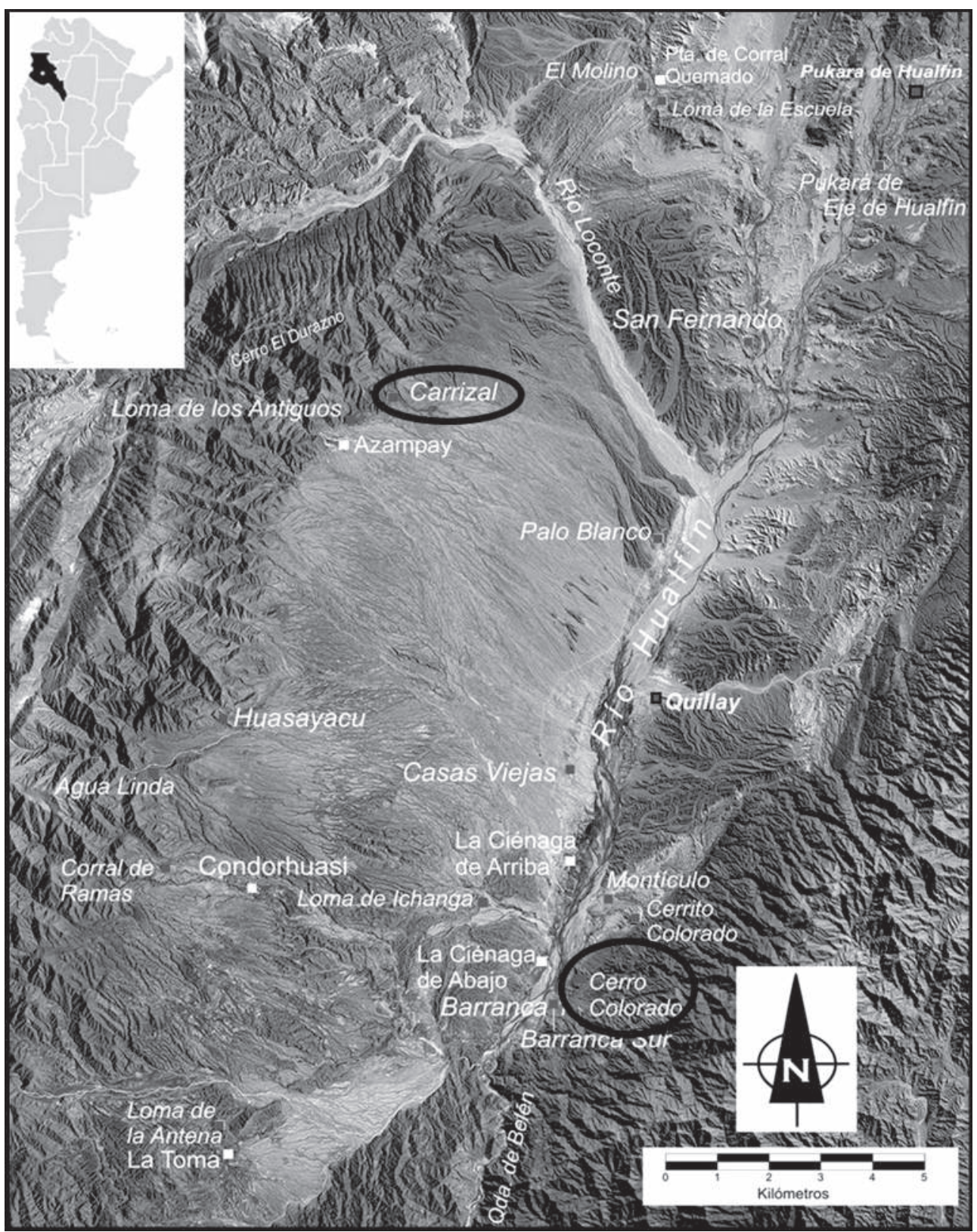

Figura 1. Mapa del valle de Hualfín (departamento de Belén, Catamarca) y ubicación de los sitios considerados. 


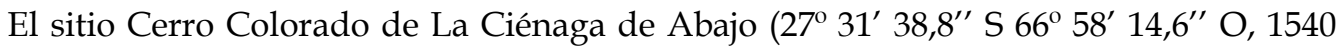
m.s.n.m) se halla en el sector sureste del valle (figura 1). Se trata de un sitio fortificado emplazado sobre un cerro de aproximadamente $150 \mathrm{~m}$ de altura, en el que se han registrado hasta el momento más de 100 estructuras distribuidas en la cima y las laderas del mismo (Wynveldt y López Mateo 2010). Hasta el momento se han excavado tres recintos en forma completa y dos parcialmente, en donde se han recuperado artefactos líticos de diferentes materias primas, tanto locales como no locales (tabla 1). Además, en este sitio se identificaron bloques de cuarzo empleados en la construcción de recintos.

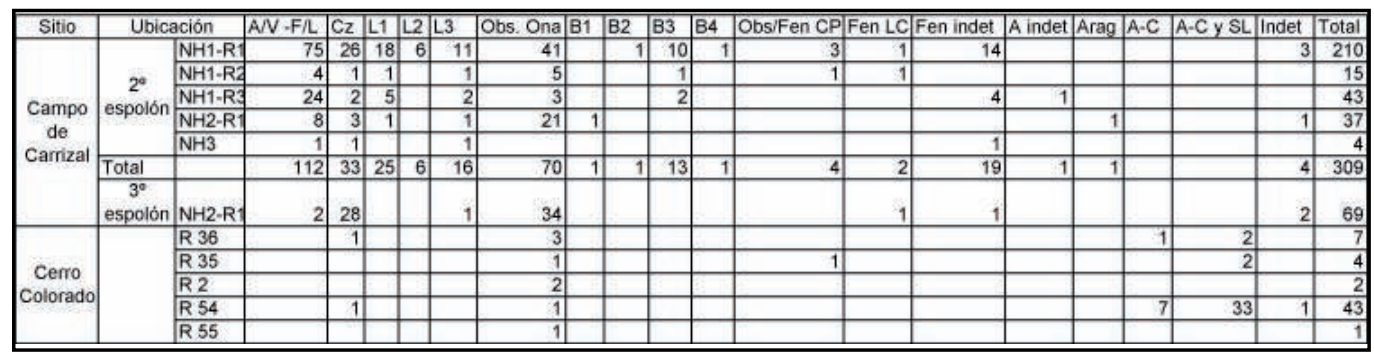

Tabla 1. Materias primas recuperadas en los sitios considerados ordenadas por recintos. Referencias: $A / V-F / L$ (arenitas y vaques feldespáticos-líticos); Cz (cuarzo); L (limolitas variedades 1, 2 y 3); Obs. Ona (obsidiana Ona); B (basaltos variedades 1, 2, 3 y 4); Obs/Fen CP (obsidiana y fenotraquitas Cueros de Purulla/Chascón); Fen LC (fenotraquita Laguna Cavi); Fen indet.

(fenotraquita indeterminada); A indet. (arenisca indeterminada); Arag (aragonita); A-C (arenita cuarzosa); A-C y SL (arenita cuarzosa y sublítica); indet. (rocas indeterminadas).

\section{Metodología}

Para el análisis de los artefactos tallados de cuarzo se siguió principalmente lo planteado por Aschero (1975, Rev. 1983) y Bellelli (1991), dividiendo el conjunto artefactual en las categorías: núcleos, desechos de talla, artefactos formatizados, artefactos no formatizados con rastros complementarios y ecofactos. Se evaluaron los siguientes atributos:

-Núcleos: dimensiones absolutas, porcentaje de corteza y designación morfológica.

-Desechos de talla: dimensiones absolutas, tipo de lasca, estado, forma de talón y porcentaje de corteza.

- Artefactos formatizados: dimensiones absolutas, forma-base, largo de filo y grupo tipológico.

- Artefactos no formatizados con rastros complementarios: dimensiones absolutas y forma-base.

- Ecofactos: dimensiones absolutas de objetos líticos recuperados en contexto arqueológico, que no presentan modificación antrópica.

\section{Análisis del material}

A continuación se presentan los resultados obtenidos a partir del estudio, los cuales se sintetizan en las tablas 2, 3, 4 y 5 .

En el sitio Campo de Carrizal se hallaron artefactos de cuarzo en estructuras del segundo y tercer espolón. En el segundo espolón se recuperaron 23 desechos de talla (NMD = 7), 1 artefacto formatizado y 2 núcleos en el recinto 1 del Núcleo habitacional 1. La mayor parte de los desechos se encuentran fragmentados, siendo escasas las lascas enteras. Se identificaron talones astillados (3) y diedros (2). Entre las formas de lascas se observaron angulares (3) y 
primaria (1), mientras que la mayoría no pudo diferenciarse (19). Las lascas enteras presentan entre 16 y $19 \mathrm{~mm}$ de longitud, 21 y $27 \mathrm{~mm}$ de ancho y 5 y $9 \mathrm{~mm}$ de espesor; los fragmentos de lascas con talón tienen entre 14 y $28 \mathrm{~mm}$ de longitud, 10 y $26 \mathrm{~mm}$ de ancho y 3 y $9 \mathrm{~mm}$ de espesor y los fragmentos de lascas sin talón presentan entre 5 y $19 \mathrm{~mm}$ de longitud, 6 a $24 \mathrm{~mm}$ de ancho y 1 y $6 \mathrm{~mm}$ de espesor. El único fragmento indiferenciado presenta 27 $\times 16 \times 15 \mathrm{~mm}$ (figura 2). También se recuperaron una punta de proyectil apedunculada de limbo triangular (figura 3) de $25 \times 12 \times 3 \mathrm{~mm}$, formatizada a partir de una forma-base lasca (pero el cuarzo empleado para su manufactura presenta inclusiones que no se observaron en los demás artefactos recuperados de esta misma materia prima, por lo cual la misma podría haber sido introducida ya terminada en el recinto) y dos núcleos de lascados aislados, sobre formas-base no diferenciadas, uno de $31 \times 33 \times 12 \mathrm{~mm}$ y otro de $49 \times 49 \times 33 \mathrm{~mm}$. Del recinto 2 del Núcleo habitacional 1 proviene 1 fragmento de lasca indiferenciada con talón (LFCT) astillado, de $19 \times 16 \times 6 \mathrm{~mm}$. En el recinto 3 del Núcleo habitacional 1 se hallaron dos desechos de talla, una lasca entera (LENT) indiferenciada con talón puntiforme de $10 \mathrm{x}$ $15 \times 2 \mathrm{~mm}$ y un fragmento longitudinal de lasca indiferenciada de $16 \times 11 \times 6 \mathrm{~mm}$ con talón liso (LFCT). Por otra parte, en el recinto 1 del Núcleo habitacional 2 se contabilizaron tres desechos de talla -una lasca angular entera (LENT) con talón liso de $24 \times 35$ × $15 \mathrm{~mm}$ con remanente de corteza (menos del 50\%), un fragmento longitudinal de lasca angular (LFCT) con talón liso de $24 \times 27 \times 10 \mathrm{~mm}$ y un fragmento de lasca angular sin talón (LFST) de 66 × $52 \times 20 \mathrm{~mm}$. Por último, en el relleno del Núcleo habitacional 3 se recuperó un fragmento de lasca indiferenciada sin talón (LFST) de $8 \times 5 \times 2 \mathrm{~mm}$. En el tercer espolón hasta el momento se cuenta con artefactos procedentes de la excavación parcial del recinto 1: entre los desechos de talla $(\mathrm{N}=23, \mathrm{NMD}=7)$ observamos que se trata mayoritariamente de fragmentos de lascas y pocas lascas enteras. Se reconocieron talones lisos (4), astillados (1) y puntiformes (1). Se registraron 10 lascas angulares, mientras más de la mitad de las lascas no pudo ser diferenciada en cuanto a su forma. Las lascas enteras presentan entre 14 y $43 \mathrm{~mm}$ de longitud, 20 y $50 \mathrm{~mm}$ de ancho y 1 y $12 \mathrm{~mm}$ de espesor; los fragmentos de lascas con talón tienen, uno de ellos $18 \times 13 \times 1 \mathrm{~mm}$ y el otro, $17 \times 10 \times 6 \mathrm{~mm}$; los fragmentos de lascas sin talón miden entre 11 y $47 \mathrm{~mm}$ de longitud, 10 y $45 \mathrm{~mm}$ de ancho y 2 y $15 \mathrm{~mm}$ de espesor y el único desecho indiferenciado tiene $29 \times 24 \times 12 \mathrm{~mm}$ (figura 4). En cuanto a los núcleos $(\mathrm{N}=2)$, uno presenta lascados aislados sobre una forma-base indiferenciada de $35 \times 45 \times 40 \mathrm{~mm}$, que posee menos de $50 \%$ de remanente de corteza, en donde las últimas lascas extraídas tienen aproximadamente de 34 a $26 \mathrm{~mm}$ de largo x 33 a $24 \mathrm{~mm}$ de ancho. En el segundo núcleo las lascas fueron extraídas mediante técnica bipolar a partir de un artefacto indiferenciado de $24 \times 17 \times 7 \mathrm{~mm}$. Por último, en este conjunto están presentes un fragmento distal de lasca de $27 \times 28 \times 16 \mathrm{~mm}$ que presenta rastros (ANFCRC) y dos ecofactos de cuarzo de $37 \times 22 \times$ $23 \mathrm{~mm}$ y $50 \times 24 \times 12 \mathrm{~mm}$.

En el Cerro Colorado se recuperaron hasta el momento tan sólo 2 artefactos de cuarzo. El procedente de la excavación del recinto 36 es un artefacto formatizado compuesto (arte-

\begin{tabular}{|c|c|c|c|c|c|c|}
\hline Sitio & DT & AF & N & ANFCRC & E & Total \\
\hline Cerro Colorado & 1 & 1 & & & & 2 \\
\hline Campo de Carrizal & 53 & 1 & 4 & 1 & 2 & 61 \\
\hline Total & 54 & 2 & 4 & 1 & 2 & 63 \\
\hline
\end{tabular}

Tabla 2. Distribución de las categorías tipológicas en los dos sitios considerados. Referencias: DT (desechos de talla); AF (artefactos formatizados); $N$ (núcleos); ANFCRC (artefactos no formatizados con rastros complementarios); E (ecofactos). 
facto mediano pequeño con bisel asimétrico oblicuo y artefacto de formatización sumaria) realizado sobre una forma-base indeterminada de $42 \times 30 \times 13 \mathrm{~mm}$. El segundo, una lasca indiferenciada sin talón (LFST) de $13 \times 21 \times 6 \mathrm{~mm}$ fue recuperado en capa en el recinto 54 .

\begin{tabular}{|c|c|c|c|c|c|c|c|c|}
\hline \multirow{2}{*}{ Sitio } & \multirow{2}{*}{\multicolumn{2}{|c|}{ Ubicación }} & \multicolumn{4}{|c|}{ Estado de fragmentación } & \multirow{2}{*}{ Total } & \multirow{2}{*}{$\%$} \\
\hline & & & LENT & LFCT & LFST & INDI & & \\
\hline \multirow{6}{*}{$\begin{array}{c}\text { Campo de } \\
\text { Carrizal }\end{array}$} & \multirow{5}{*}{$2^{\circ}$ espolón } & NH1-R1 & 3 & 4 & 15 & 1 & 23 & 43 \\
\hline & & NH1-R2 & & 1 & & & 1 & 2 \\
\hline & & NH1-R3 & 1 & 1 & & & 2 & 4 \\
\hline & & NH2-R1 & 1 & 1 & 1 & & 3 & 6 \\
\hline & & $\mathrm{NH} 3$ & & & 1 & & 1 & 2 \\
\hline & $3^{\circ}$ espolón & NH2-R1 & 5 & 2 & 15 & 1 & 23 & 43 \\
\hline Total & & & 10 & 8 & 33 & 2 & 53 & 100 \\
\hline $\begin{array}{c}\text { Cerro } \\
\text { Colorado }\end{array}$ & \multicolumn{2}{|c|}{ Recinto 54} & & & 1 & & 1 & 100 \\
\hline Total & & & & & 1 & & & 100 \\
\hline
\end{tabular}

Tabla 3. Estado de fragmentación de los desechos de talla por sitio y recintos. Referencias: LENT (lascas enteras); LFCT (lascas fracturadas con talón); LFST (lascas fracturadas sin talón); INDI (desechos indiferenciados).

\begin{tabular}{|c|c|c|c|c|c|c|c|}
\hline \multirow{2}{*}{ Sitio } & \multirow{2}{*}{\multicolumn{2}{|c|}{ Ubicación }} & \multicolumn{3}{|c|}{ Formas de lascas } & \multirow{2}{*}{ Total } & \multirow{2}{*}{$\%$} \\
\hline & & & Angular & Primaria & Indiferenciada & & \\
\hline \multirow{6}{*}{$\begin{array}{c}\text { Campo de } \\
\text { Carrizal }\end{array}$} & \multirow{5}{*}{$2^{\circ}$ espolón } & NH1-R1 & 3 & 1 & 19 & 23 & 43 \\
\hline & & NH1-R2 & & & 1 & 1 & 2 \\
\hline & & NH1-R3 & & & 2 & 2 & $\overline{4}$ \\
\hline & & NH2-R1 & 3 & & & 3 & $\overline{6}$ \\
\hline & & $\mathrm{NH} 3$ & & & 1 & 1 & 2 \\
\hline & $3^{\circ}$ espolón & NH2-R1 & 10 & & 13 & 23 & 43 \\
\hline Total & & & 16 & 1 & 36 & 53 & 100 \\
\hline $\begin{array}{c}\text { Cerro } \\
\text { Colorado }\end{array}$ & \multicolumn{2}{|c|}{ Recinto 54} & & & 1 & 1 & 100 \\
\hline Total & & & & & 1 & & 100 \\
\hline
\end{tabular}

Tabla 4. Formas de lascas por sitio y recintos.

\begin{tabular}{|c|c|c|c|c|c|c|c|c|c|}
\hline \multirow{2}{*}{ Sitio } & \multirow{2}{*}{\multicolumn{2}{|c|}{ Ubicación }} & \multicolumn{5}{|c|}{ Formas de talones } & \multirow{2}{*}{ Total } & \multirow{2}{*}{$\%$} \\
\hline & & & Liso & Astillado & Diedro & Puntiforme & No Diferenciado & & \\
\hline \multirow{6}{*}{$\begin{array}{l}\text { Campo } \\
\text { de } \\
\text { Carrizal }\end{array}$} & \multirow{5}{*}{$2^{\circ}$ espolón } & NH1-R1 & & 3 & \begin{tabular}{|l|}
2 \\
\end{tabular} & & \begin{tabular}{|c|}
1 \\
\end{tabular} & 6 & 33 \\
\hline & & NH1-R2 & & 1 & & & & 1 & 6 \\
\hline & & NH1-R3 & 1 & & & 1 & & 2 & 11 \\
\hline & & NH2-R1 & 2 & & & & & 2 & 11 \\
\hline & & NH3 & & & & & & & \\
\hline & $3^{\circ}$ espolón & NH2-R1 & 4 & 1 & & 1 & 1 & 7 & 39 \\
\hline Total & & & 7 & 5 & 2 & 2 & 2 & 18 & 100 \\
\hline
\end{tabular}

Tabla 5. Formas de talones de los desechos de talla de Campo de Carrizal, por recintos. 


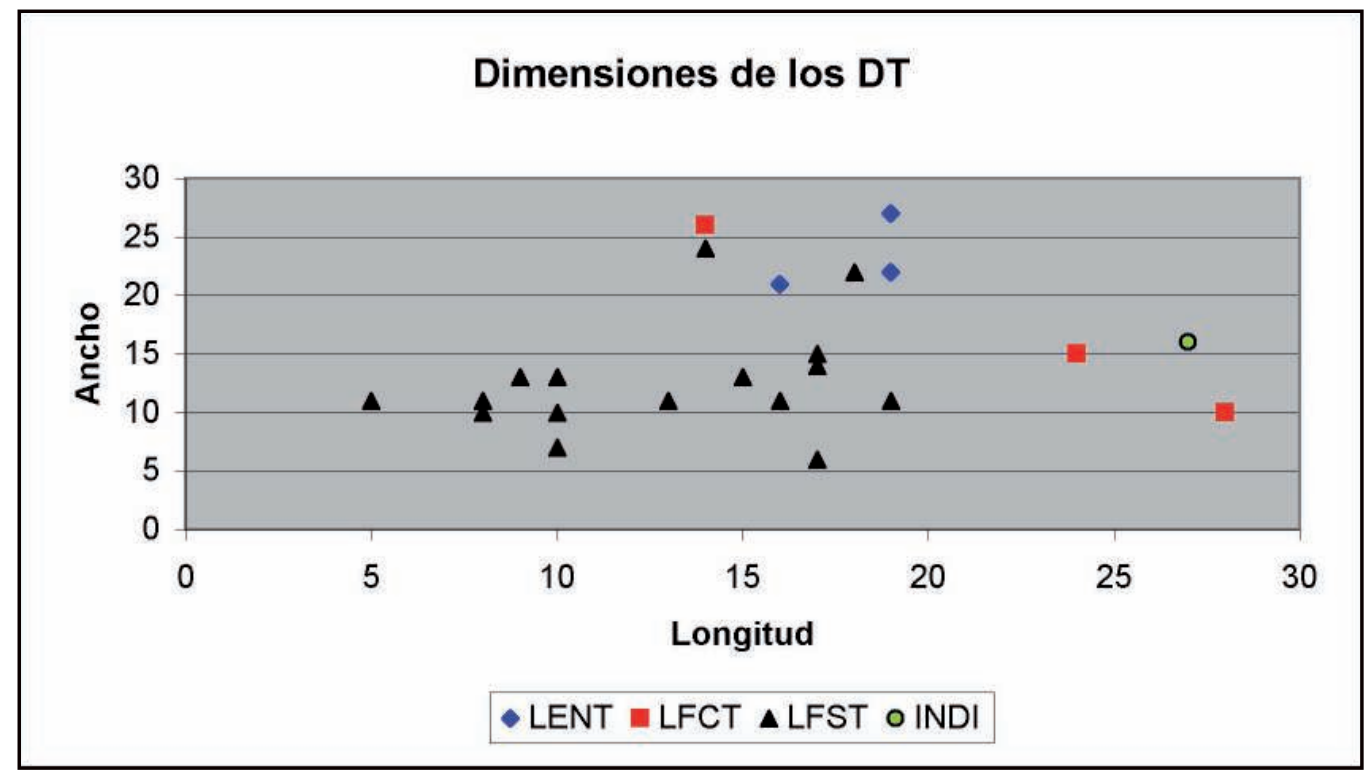

Figura 2. Dimensiones de los desechos de talla diferenciados por estado de fragmentación, correspondientes al NH1-R1 (2espolón) de Campo de Carrizal.

\section{Discusión y conclusiones}

En el valle de Hualfín, el cuarzo constituye una materia prima de carácter local. En el sitio Cerro Colorado observamos que la misma fue utilizada tanto para la manufactura de artefactos como para la construcción. A partir de la mayor cantidad de piezas recuperadas en el sitio Campo de Carrizal, podemos obtener información sobre aspectos relacionados con la explotación de esta materia prima. En líneas generales plantemos que es posible que el cuarzo haya sido introducido al sitio bajo la forma de bloques irregulares y/o, en menor medida, de cantos rodados o parcialmente rodados, que fueron explotados a partir de plataformas naturales sin preparación alguna a través de talla directa con percutor duro. A sostener esto contribuyen las evidencias sobre etapas iniciales de la secuencia de reducción representadas en el sitio. Se trata de los núcleos de lascados aislados y lascas angulares e indiferenciadas tanto enteras como fragmentadas, que se vinculan a estadios iniciales por sus dimensiones, el alto porcentaje de talones lisos y la presencia de corteza sobre sus superficies. Es importante destacar que se ha hallado un núcleo vinculado a la talla bipolar, pero se ignoran aún las razones de la

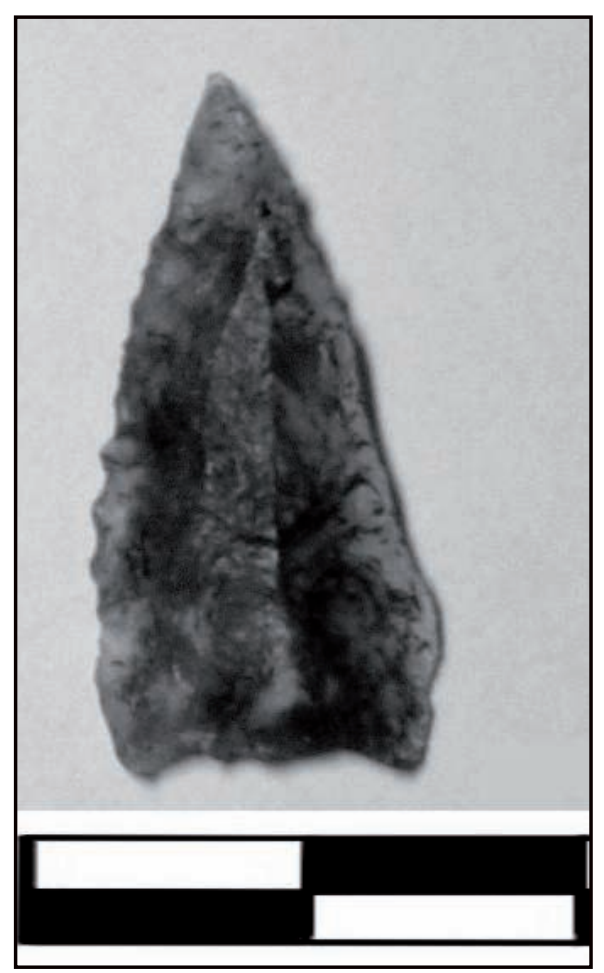

Figura 3. Punta de proyectil apedunculada de cuarzo recuperada en el sitio Campo de Carrizal. 


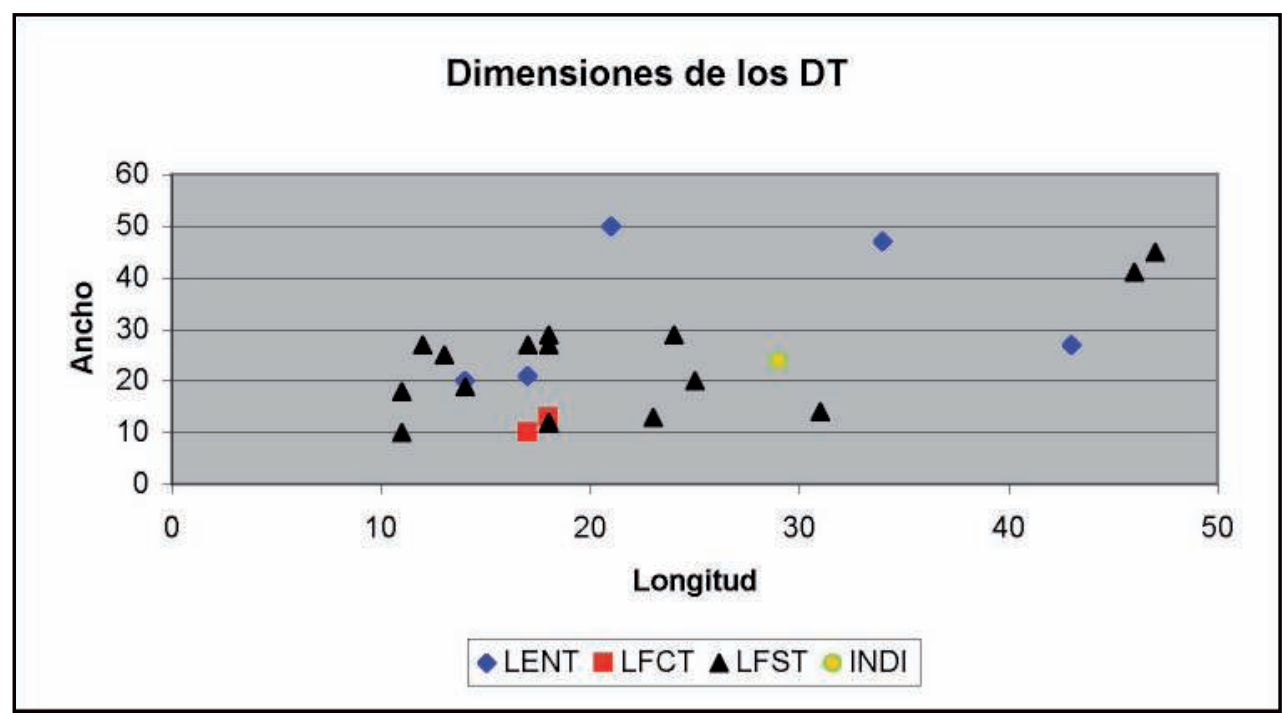

Figura 4. Dimensiones de los desechos de talla diferenciados por estado de fragmentación, recuperados en el NH2-R1 (3ºspolón) de Campo de Carrizal.

utilización de esta técnica sobre esta materia prima. En cuanto a los artefactos formatizados planteamos que, por sus características, no parecen haber demandado mucho tiempo para su confección y en cuanto a la punta de proyectil, además, que la misma pudo haber sido introducida al sitio y/o recinto ya terminada. Por último, los desechos atribuidos a etapas más avanzadas de la reducción son escasos, estando representados por muy pocas lascas de tamaños muy pequeños o cercanos a los $10 \mathrm{~mm}$ y por talones puntiformes.

El empleo del cuarzo como materia prima para la talla también ha sido mencionado en otros sitios del Noroeste argentino temporalmente relacionados con los sitios aquí tratados. Elías (2010) indica el hallazgo de artefactos de cuarzo en sitios del departamento de Antofagasta de la Sierra (Catamarca) y Gaál (2011) se refiere al empleo del cuarzo en el sitio Rincón Chico (valle de Yocavil) tanto para fines constructivos como para la manufactura de artefactos. Siempre se trata de un recurso local, que aparece en los registros en muy baja proporción en relación a otras materias primas -a excepción de Rincón Chico-, se observa un predominio de los desechos de talla en los conjuntos y no se menciona vinculación con talla bipolar como hemos indicado nosotros.

Si bien no disponemos de evidencia que nos permita acercarnos a la variabilidad de artefactos formatizados en cuarzo entre nuestros hallazgos, Gaál (2011) menciona para Rincón Chico la recuperación de raederas, cuchillos y lascas con retoque sumario e indica el uso de filos naturales en esta materia prima. Por otra parte, la punta de proyectil apedunculada de cuarzo recuperada en Campo de Carrizal es similar a otras puntas de cuarzo que se encuentran depositadas en el Museo Arqueológico Condor Huasi de Belén (Catamarca), las cuales fueron recuperadas en el valle de Hualfín, pero que carecen de referencia espacio-temporal precisa. Es importante señalar en este punto que en los dos sitios considerados aquí, también se recuperaron puntas de proyectil triangulares apedunculadas en otras materias primas (obsidiana y fenotraquita). 
Finalmente, ocurre que en general observamos que el cuarzo, a pesar de ser una materia prima local, aparece en los sitios en muy bajas proporciones en relación con otras materias primas locales, tendencia que parecería repetirse en otros sitios del Noroeste argentino adscriptos al mismo lapso temporal que nos ocupa.

Con este trabajo hemos intentado dar un primer paso en la caracterización de cuarzo como materia prima en el valle de Hualfín durante el Período de Desarrollos Regionales/ Inka, cuestión que deberá profundizarse en un futuro próximo.

\section{Agradecimientos}

A la Dra. N. Franco por los valiosos comentarios y sugerencias y al Dr. F. Wynveldt por el procesamiento de las imágenes. Todo lo vertido en este trabajo es exclusiva responsabilidad de la autora.

\section{Bibliografía citada}

Aschero, C.

1975 Ensayo para una clasificación morfológica de artefactos líticos aplicada a estudios tipológicos comparativos. Informe presentado al CONICET.

1983 Ensayo para una clasificación morfológica de artefactos líticos. Apéndices A y B. Cátedra de Ergología y Tecnología. Universidad de Buenos Aires. Manuscrito.

Bellelli, C.

1991 Los desechos de talla en la interpretación arqueológica. Un sitio de superficie en el Valle de Piedra Parada (Chubut). Shincal 3 (2): 79-93.

Cortegoso, $\mathrm{V}$.

2008 Disponibilidad de recursos líticos en el noroeste de Mendoza: cambios en la organización tecnológica en la cuenca del río Blanco. Cazadores Recolectores en el Cono Sur 3: 95-113.

Dana, E. y O. Hurlbut

1956 Manual de Mineralogía, Editorial Reverté.

Elías, A.

2010 Estrategias tecnológicas y variabilidad de los conjuntos líticos de las sociedades Tardías en Antofagasta de la Sierra (Provincia de Catamarca, Puna Meridional Argentina). Tesis para optar al grado de Doctor de la Universidad de Buenos Aires. Área Arqueología.

Gaál, E.

2011 Un acercamiento preliminar al estudio de la base local de recursos líticos y al análisis artefactual en el poblado arqueológico de Rincón Chico (período Tardío), valle de Yocavil, Catamarca. Comechingonia Virtual (1):1-38.

González Bonorino, F. 1972 Descripción Geológica de la Hoja 13c, Fiambalá. Boletín nº 127. Ministerio de Industria y Minería. Subsecretaria de Minería. Dirección Nacional de Geología y Minería, Buenos Aires.

Ruíz Huidobro, O.

1975 Descripción geológica de la hoja 12c, Laguna Helada. Provincia de Catamarca. Boletín $n^{\circ}$ 146, Ministerio de Economía, Secretaria de Estado de Recursos Naturales y Ambiente Humano, Subsecretaria de Minería. Servicio Geológico Nacional, Buenos Aires. 
Sempé de Gómez Llanes, M. C.

1981 Investigaciones arqueológicas en el departamento de Belén (Catamarca). Novedades del Museo de La Plata 1(2):19.

Wynveldt, F.

2009 La Loma de los Antiguos de Azampay, un sitio defensivo del Valle de Hualfin (Catamarca, Argentina). Sociedad Argentina de Antropología, Buenos Aires.

Wynveldt, F. y M. López Mateo

2010 Pueblos protegidos, murallas y divisaderos: un paisaje arqueológico defensivo en La Ciénaga. Aldeas protegidas, conflicto y abandono. Investigaciones arqueológicas en La Ciénaga (Catamarca, Argentina), (ed. por B. Balesta y N. Zagorodny), pp. 277-312. Ediciones Al Margen, La Plata.

Zagorodny, N., E. Rivera y C. Valencia

2007 Análisis antracológico y objetos de madera del sitio Campo de Carrizal. Actas del XVI Congreso Nacional de Arqueología Argentina (1):133-139. Jujuy. 